\title{
Bio-Based Polyester/Cellulose Films for Engineering Applications ${ }^{+}$
}

\author{
Elena-Ruxandra Radu, Denis Mihaela Panaitescu, Augusta Raluca Gabor, Cristian Andi Nicolae \\ and Adriana Nicoleta Frone *
}

\begin{abstract}
National Institute for Research\&Development in Chemistry and Petrochemistry-ICECHIM, 202 Spl.
Independenței, 060021 Bucharest, Romania; radu.elena.ruxandra@gmail.com (E.-R.R.);

denisspan02@yahoo.com (D.M.P.); ralucagabor@yahoo.com (A.R.G.); ca_nicolae@yahoo.com (C.A.N.)

* Correspondence: adriana.frone@icechim.ro

+ Presented at the 16th International Symposium "Priorities of Chemistry for a Sustainable Development" PRIOCHEM, Bucharest, Romania, 28-30 October 2020.
\end{abstract}

Published: 11 November 2020

Keywords: Poly(lactic acid); Poly(hydroxybutyrate); nanocellulose; nanocomposites

A large part of the materials intended for engineering applications are still based on non-renewable resources, thus contributing to the accumulation of plastic wastes [1]. The replacement of petroleum-based polymers with bio-based ones is a must of the current period. Poly(lactic acid) (PLA) is the most used biopolymer due to its many advantages arising from its good mechanical properties and transparency along with the availability on the market, and easy processing [2]. Poly(3-hydroxybutyrate) (PHB) is one of the most promising biopolyesters obtained from bacterial fermentation and is seen as a substitute of petroleum-based polymers in biomedical and engineering applications [3]. Nanocellulose represents a renewable and innovative filler, that incorporated or combined with other biopolymers may give rise to a multitude of performing and sustainable multifunctional materials $[1,3]$. This work aimed to obtain PLA/PHB composite films reinforced with different concentrations $(0-4 \mathrm{wt} \%)$ of nanocellulose extracted from cheap sources. Nanocellulose extracted from agricultural waste (NC), polylactic acid (PLA 4032 D), in the form of pellets, containing 1.4\% D isomer and with an average molecular weight of $220 \mathrm{kDa}$ (NatureWorks LLC, Minnetonka, MN, USA) and PHB powder from Biomer (Germany) were used in this study. The polymer composites were obtained by melt-mixing method using a fully automated Brabender plastograph and pressed into films with $0.28 \mathrm{~mm}( \pm 0.001 \mathrm{~mm})$ thickness. Incorporation of NC in the PLA/PHB matrix influenced the Young's modulus (Y), which was improved in relation to the control sample regardless NC concentration (Figure 1). The thermal stability of the obtained composites was not diminished by the addition of NC. Moreover, an increase in the onset temperature by $9^{\circ} \mathrm{C}$ was attained in the composite samples containing the highest NC amount. DMTA results showed an enhancement of the storage modulus for all PLA/PHB/NC composites, especially at room temperature, revealing the reinforcing effect of this type of fibers.

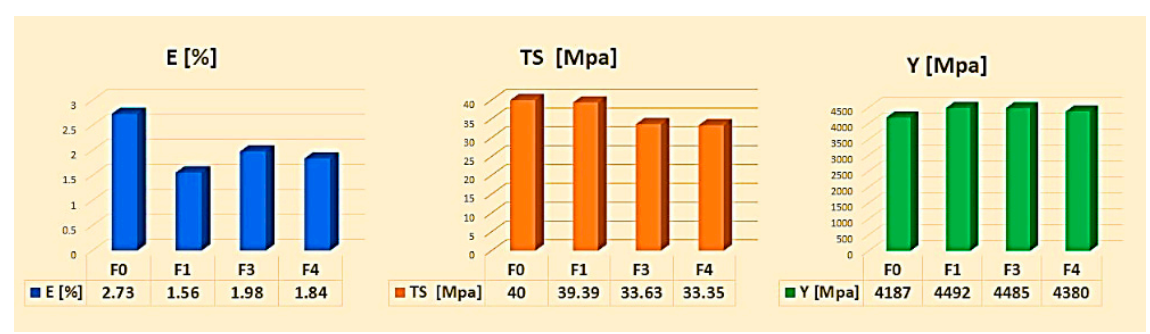

Figure 1. Mechanical properties of PLA/PHB matrix and PLA/PHB/NC composites. 
Polyesters-nanocellulose composite films intended for engineering applications were obtained using only bio-based materials. TGA analysis highlighted the positive effect of NC fillers on improving the thermal stability of the PLA/PHB matrix. Both static tensile test and dynamical mechanical analysis pointed out an improved stiffness upon the incorporation of NC. Thus, the obtained composites can be considered as potential candidates for safe toys.

Acknowledgments: This work was supported by a grant of the Romanian Ministryof Education and Research, CNCS-UEFISCDI, project number PN-III-P1-1.1-TE-2019-1333, no. 67/2020-FLEX4PLA, within PNCDI III.

\section{References}

1. Arrieta, M.P.; Fortunati, E.; Dominici, F.; López, J.; Kenny, J.M. Bionanocomposite films based on plasticized PLA-PHB/cellulose nanocrystal blends. Carbohydr. Polym. 2015, 121, 265-275.

2. Frone, A.N.; Berlioz, S.; Chailan, J.-F.; Panaitescu, D.M. Morphology and thermal properties of PLA-cellulose nanofibers composites. Carbohydr. Polym. 2013, 91, 377-384.

3. Panaitescu, D.M.; Nicolae, C.A.; Gabor, A.R.; Trusca, R. Thermal and mechanical properties of poly(3-hydroxybutyrate) reinforced with cellulose fibers from wood waste. Ind. Crops Prod. 2020, 145, 112071.

Publisher's Note: MDPI stays neutral with regard to jurisdictional claims in published maps and institutional affiliations.

(C) 2020 by the authors. Licensee MDPI, Basel, Switzerland. This article is an open access article distributed under the terms and conditions of the Creative Commons Attribution (CC BY) license (http://creativecommons.org/licenses/by/4.0/). 